\title{
Hexagonal ice in pure water and biological NMR samples
}

\section{Journal Article}

Author(s):

Bauer, Thomas; Gath, Julia; Hunkeler, Andreas; Ernst, Matthias (D); Böckmann, Anja; Meier, Beat H.

Publication date:

2017-01

Permanent link:

https://doi.org/10.3929/ethz-b-000129585

Rights / license:

In Copyright - Non-Commercial Use Permitted

Originally published in:

Journal of Biomolecular NMR 67(1), https://doi.org/10.1007/s10858-016-0080-7

Funding acknowledgement:

159707 - NMR studies in the Solid State (SNF)

146757 - NMR studies in the Solid State (SNF) 


\title{
Hexagonal Ice in Pure Water and Biological NMR Samples
}

Thomas Bauer ${ }^{1}$, Julia Gath ${ }^{1}$, Andreas Hunkeler ${ }^{1}$, Matthias Ernst*1, Anja Böckmann*2, Beat H. Meier*1

(1) ETH Zurich, Physical Chemistry, Vladimir-Prelog-Weg 2, CH-8093 Zurich, Switzerland

(2) Institut de Biologie et Chimie des Protéines, UMR 5086 CNRS, Université de Lyon 1, 7 passage du Vercors, 69367 Lyon, France

\begin{abstract}
Ice, in addition to "liquid" water and protein, is an important component of protein samples for NMR spectroscopy at subfreezing temperatures but it has rarely been observed spectroscopically in this context. We characterize its spectroscopic behavior in the temperature range from $100 \mathrm{~K}$ to $273 \mathrm{~K}$, and find that it behaves like pure water ice. The interference of magic-angle spinning (MAS) as well as rf multiple-pulse sequences with Bjerrum-defect motion greatly influences the ice spectra.
\end{abstract}

\section{Introduction}

Water plays a vital role in virtually all biological processes and its interaction with proteins is essential for their structure, stability, internal dynamics and folding landscape (Lynden-Bell et al. 2010; Bagchi 2013). Although most biological processes take place In the presence of liquid water at temperatures above the freezing point, there is a considerable interest in the properties of proteins at low temperature. A related field of particular interest is the characterization of the role of anti-freeze proteins which prevent freezing of water inside the cells in organisms living at subfreezing temperatures (Siemer and McDermott 2008; Siemer et al. 2010).

For biological solid-state NMR, low temperature measurements are interesting in particular for signal enhancement by Boltzmann population effects, dynamic nuclear polarization (Barnes et al. 2008; Can et al. 2015; Akbey and Oschkinat 2016) or to reduce the dynamics in proteins (Havlin and Tycko 2005). A frozen protein sample usually consists of three components: the protein, "liquid" water forming a layer around the protein and solid ice (Siemer et al. 2010). The protein and the water phases as well as the exchange of 
polarization between them have been described in detail (Lesage and Böckmann 2003; Böckmann et al. 2005; Lesage et al. 2008; Böckmann et al. 2009; Siemer et al. 2010; Siemer et al. 2012) while the ice resonance is usually not observed in biomolecular NMR experiments.

In ice, under ambient pressure and temperature conditions, water molecules form a crystalline solid with a hexagonal symmetry. Following the Bernal-Fowler building rules (Bernal and Fowler 1933), each oxygen atom is coordinated tetrahedrally by four neighboring oxygen atoms with one hydrogen atom in between them to form a hydrogen bond. Violations of these building rules can occur in the form of so called Bjerrum defects (see Figure 1a), where either two protons (D-defect) or no protons (L-defect) are located between two given oxygen atoms.
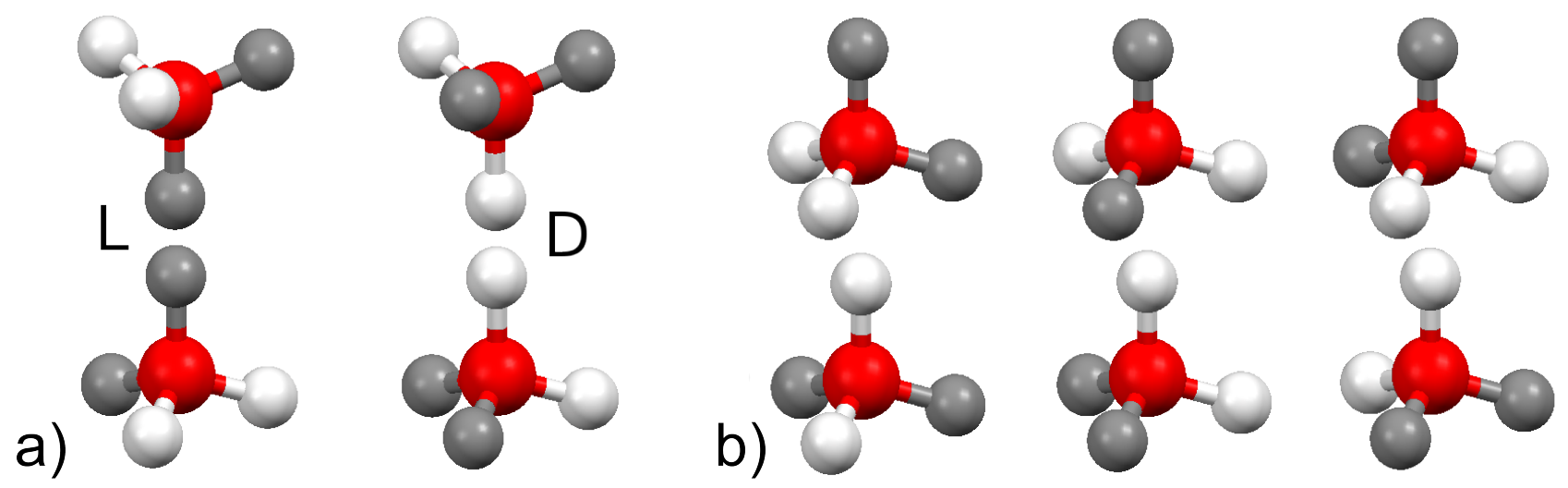

Fig. 1: a) Bjerrum L- and D- defects as they occur in the crystal lattice. b) The six possible orientations of the water molecule within the tetrahedral arrangement of the oxygen atoms. The positions occupied by protons are marked in white, unoccupied but possible positions in gray.

Although the water molecule is translationally restricted to its lattice site, the tetrahedral grid allows six possible orientations of the molecule's protons around their central oxygen atom (Figure 1b). The rate constant at which those reorientations occur is temperature dependent and also depends on the number of defects. (Fujara et al. 1988) The typical number of defects in the lattice is big enough to create a significant effect on the NMR properties of ice, for example the longitudinal spin relaxation. The reorientation process has been characterized before (Barnaal and Lowe 1968; Siegle and Weithase 1969; Geil et al. 2005; Ba et al. 
2011) and was found, for pure ice, to follow an Arrhenius-type rate law with an activation energy of $56.5 \mathrm{~kJ} / \mathrm{mol}$ and a pre-exponential factor in the order of $10^{15}$. The range of expected rate constants thus reaches from $6 \mathrm{~s}^{-1}$ at $200 \mathrm{~K}$ to $6 \cdot 10^{4} \mathrm{~s}^{-1}$ at $270 \mathrm{~K}$, which is comparable to the dipolar ${ }^{1} \mathrm{H}-{ }^{1} \mathrm{H}$ coupling constant of roughly $43 \mathrm{kHz}$ for an average intramolecular proton distance of approximately $1.4 \AA$ in ice (Bernal and Fowler 1933). Therefore, the motion has considerable effects on the NMR spectra of pure ice.

In the following, we study ice in pure water sample and as a component of a frozen HET-s (218-289) protein sample in the temperature range from $100 \mathrm{~K}$ to $273 \mathrm{~K}$. The protein HET-s (218-289), constitutes the prionforming domain of the HET-s protein from the fungus Podospora anserina. In its fibrillar amyloid form, HET-s (218-289) is a left handed $\beta$-solenoid with two windings formed by one molecule (Wasmer et al. 2008; van Melckebeke et al. 2010) (Wasmer et al. 2009b). In the NMR rotor, HET-s(218-289) fibrils form a densely packed and highly viscous protein sample with a water contents of roughly $75 \%$ by mass as determined by the weight loss upon lyophillization. It can act as a model for many biomolecular fibrillar samples. Furthermore, we characterize the chemical exchange between the water and ice phases.

\section{Materials and Methods}

\subsection{Second Momentum and Spin-diffusion Rate Calculations}

In first approximation, the NMR line width in a simple one-pulse experiment of a static sample of ice is governed by the dipolar coupling between the protons. An estimate of the resulting line width can be given by determining the powder average of the van Vleck second moment $\left\langle\Delta \omega^{2}\right\rangle$ (van Vleck 1948; Slichter 1978)

$$
\left\langle\Delta \omega^{2}\right\rangle=\frac{3}{5}\left(\frac{\mu_{0}}{4 \pi}\right)^{2} \gamma^{4} \hbar^{2} I(I+1) \sum_{k} \frac{1}{r_{j k}^{6}}
$$

with $\boldsymbol{\mu}_{\mathbf{0}}, \boldsymbol{\gamma}$ and $\hbar$ being the usual constants, $I=\mathbf{1} / \mathbf{2}$ and $\boldsymbol{j}$ the index for the observed spin. The index $\boldsymbol{k}$ runs over all the neighboring spins, with the internuclear distance $\boldsymbol{r}_{\boldsymbol{j} \boldsymbol{k}}$. Assuming a Gaussian line shape the full width at half height, $\Delta v$, in frequency units is then given by 


$$
\Delta v=2 \sqrt{2 \ln 2\left\langle\Delta \omega^{2}\right\rangle} / 2 \pi
$$

The two relations will be used in the following sections to compute the estimated line width of the dipolarbroadened spectrum in the case of very low temperatures, i.e. the quasi-rigid case, and for high temperatures with rapid reorientation.

A rough estimate for the proton spin-diffusion rate constant can be obtained using the perturbation description of Chapter V of Abragams book. (Abragam 1961). The probability of a spin flip is estimated as $\boldsymbol{W} \approx \sqrt{\left\langle\boldsymbol{\Delta \boldsymbol { \omega } ^ { 2 } \rangle}\right.} / \mathbf{3 0}$, and the spin diffusion rate constant by $\boldsymbol{D}=\boldsymbol{W} \boldsymbol{a}^{2}$, where $\boldsymbol{a}$ is the average distance between neighboring protons which is, for the high-temperature ice (fast defect motion), $2.73 \AA$. For this order-of-magnitude estimation, the effect of magic-angle spinning is neglected, except that for the estimation of the second moment the linewidth of the central line under MAS is taken into account. For an experimental linewidth of $\Delta v=\mathbf{1 0} \mathbf{k H z}$ this leads to $D \approx \mathbf{7} \cdot \mathbf{1 0}^{-17} \mathrm{~m}^{2} / \mathrm{s}$. From this value, the diffusion length in a time interval $t$ can be calculated as $\langle l\rangle=\sqrt{6 D t}$, which, for $\mathrm{t}=2000 \mathrm{~s}$ leads to $\langle l\rangle \approx 1 \mu \mathrm{m}$.

\subsection{Dynamic Simulation of a Two-Spin System}

The six-site jump model of Figure $1 \mathrm{~b}$ can be employed to predict the effects of rapid reorientational dynamics on the NMR signal of ice. The time evolution of a dynamic two-proton spin system with CSA and dipolar interaction was calculated by implementing the six-site jump model using an exchange-based algorithm using the program GAMMA (Smith et al. 1994). All possible jumps between the six conformations were considered to possess equal probabilities.

\subsection{Sample Preparation}

For all experiments on pure water, polycrystalline hexagonal ice was prepared from deionized water with a resistance of $18 \mathrm{M} \Omega$. It was gradually frozen in the NMR probe using either chilled nitrogen gas or a commercial Bruker cooling unit (BCU), depending on the temperature region the experiments were conducted in. Attempts of more rapid sample freezing in liquid nitrogen or methanol chilled with dry ice showed little to no effect on the experimental results. For the deuterated sample, deuterium enriched water 
(99.8\% D, purchased from Sigma Aldrich) was used.

Furthermore, MAS experiments were performed on ${ }^{2} \mathrm{H},{ }^{13} \mathrm{C},{ }^{15} \mathrm{~N}$-labeled HET-s(218-289) with a C-terminal His 6 tag. Protein expression and fibrillization were done as described in (van Melckebeke et al. 2011). The fibrillization was performed in $\mathrm{H}_{2} \mathrm{O}$ at $\mathrm{pH} 7.5$ and, consequently, the amide and exchangeable side-chain protons are fully back exchanged with ${ }^{1} \mathrm{H}$. The protein was centrifuged for $45 \mathrm{~min}$ at $134,000 \mathrm{~g}$ into Bruker $1.3 \mathrm{~mm}$ rotors using a home-made filling tool (Böckmann et al. 2009). The highly concentrated and viscous pellet was frozen inside the NMR probe before starting MAS rotation to avoid additional centrifugation and the formation of a supernatant phase (Böckmann et al. 2009; Bertini et al. 2011; Böckmann et al. 2015). The resulting samples are heterogenous on a mesoscopic scale with single fibrils as well as clustered protein fibril domains but homogenous in terms of the local structure. (Wasmer et al. 2009a). They contain roughly $75 \%$ water (weight/weight) or $3 \mathrm{~g}$ water per gramm of protein, some of which might still be supernatant. (Böckmann et al. 2009). Upon freezing, 70\% of the water is transformed to ice (Bauer et al. 2016\} leaving 0.9 g/g. Here no contribution from supernatant water is expected as it is frozen. This remaining water progressively solidifies upon further cooling until it becomes undetectable below $220 \mathrm{~K}$. The amount of hydration layer in a globular protein has been estimated between 0.2 and $0.5 \mathrm{~g} / \mathrm{g}$ protein. (Jeffrey and Saenger 1994)

\subsection{NMR experiments}

Static experiments were recorded at a proton resonance frequency of $300 \mathrm{MHz}$ using a radio-frequency (RF) amplitude of $100 \mathrm{kHz}$. A practical problem with the detection of ice protons by NMR is their long longitudinal relaxation time, while the transverse decay time is very short. $\boldsymbol{T}_{\mathbf{1}}$ at a field strength of $7 \mathrm{~T}$ and a temperature of $240 \mathrm{~K}$, for example, is in the order of $1000 \mathrm{~s}$ while the transverse magnetization decays within a few microseconds in static experiments (Siegle and Weithase 1969). Additionally, the large proton background of standard MAS probes, such as protonated end-caps and drive-tips of the MAS rotors, can interfere with the signal to be observed. To mitigate this problem, a $2.5 \mathrm{~mm}$ Chemagnetics MAS probe was customized to minimize the proton background. All protonated stator material was removed and replaced by Teflon. The 
quality factor of the probe was then artificially lowered by detuning the RF circuit to smaller than 100 , leading to a significant reduction of the experimental dead time to roughly $6 \mu \mathrm{s}$.

MAS experiments were performed on pure $\mathrm{H}_{2} \mathrm{O}$ as well as deuterated and back-exchanged HET-s (218-289) with a $1.3 \mathrm{~mm}$ Bruker low-temperature probe. The spectra were recorded at a spinning frequency of $40 \mathrm{kHz}$ and a proton resonance frequency of $600 \mathrm{MHz}$ and a $\mathrm{RF}$ amplitude of $100 \mathrm{kHz}$ in the same temperature range as for the static experiments. Spectra consist of a single scan, acquired after a waiting time of $12 \mathrm{~h}$ to guarantee thermal equilibration (and spin relaxation) and a further scan was acquired $3 \mathrm{~s}$ later to subtract the fast-relaxing component.

A windowed MSHOT-3 sequence (Hohwy and Nielsen 1997) was used for homonuclear decoupling experiments using a $1.3 \mathrm{~mm}$ Bruker MAS probe and a RF amplitude of $150 \mathrm{kHz}$. The measurements were conducted at a proton frequency of $400 \mathrm{MHz}$ on a static ice and a deuterated and back exchanged sample of HET-s(218-289). Spectra consist of a single scan, acquired after a waiting time of between $12 \mathrm{~h}$ and $18 \mathrm{~h}$ to guarantee thermal equilibration (and spin relaxation) and a further scan was acquired $3 \mathrm{~s}$ later to subtract the fast-relaxing component.

Single-scan spectra on a deuterated ice sample (observing the residual protons) were recorded on a $1.3 \mathrm{~mm}$ Bruker MAS probe at $10 \mathrm{kHz}$ MAS at a proton resonance frequency of $400 \mathrm{MHz}$ and a RF amplitude of $100 \mathrm{kHz}$ under deuterium decoupling using TPPM with $100 \mathrm{kHz}$ rf amplitude, a pulse length of $4.8 \mu \mathrm{s}$ and a phase angle of $15^{\circ}$. Again a single scan was recorded after $12 \mathrm{~h}$ equilibration.

\section{Results and Discussion}

Figure 2a shows the proton spectra acquired in single-pulse experiments on polycrystalline hexagonal ice. The line shape changes with decreasing temperatures from a mostly Gaussian line shape with a full line width at half height of approximately $28 \mathrm{kHz}$ at $260 \mathrm{~K}$ to a more complex line shape with an emerging plateau and a line width of roughly $88 \mathrm{kHz}$ at the lowest temperature of $130 \mathrm{~K}$. Figure 2 also shows a simulation carried out in GAMMA including the ten closest protons in a fully rigid hexagonal ice lattice. 
The simulated rigid-lattice spectrum agrees well with the experimental spectrum at $130 \mathrm{~K}$ in terms of the line width as well as the qualitative line shape and explains the emerging line shape at low temperatures. The differences between the simulation and the low temperature experiment can be attributed to the limited number of spins used for the simulation. Based on Eqs. (1) and (2), one can estimate the expected line width $\Delta v$ in the rigid lattice to be $82 \mathrm{kHz}$, in agreement with the experimentally determined line width of $88 \mathrm{kHz}$ at the lowest temperature. The $130 \mathrm{~K}$ spectrum can, therefore, be understood as mostly dipolar broadened with little dynamics.

The narrowing in the line shape with increasing temperature indicates the onset of dynamics. In the fastexchange limit, intramolecular interactions vanish but intermolecular ones partially remain. Simulating this process in a large spin system is difficult due to computational limitations. However, a crude estimate of the resulting line width can be obtained by setting both protons of $\mathrm{H}_{2} \mathrm{O}$ in the center of the tetrahedral grid they are moving on, which corresponds to the oxygen position in the hexagonal lattice and neglecting the intramolecular dipolar coupling. Using Eqs. (1) and (2), the calculated line width yields a value $\Delta \boldsymbol{v}$ of $20 \mathrm{kHz}$, in agreement with the line width observed in the experiment close to the melting temperature. The high temperature spectrum at $260 \mathrm{~K}$ can, therefore, be understood by the partially averaged intermolecular dipolar couplings. The range of measurements between 130 and $260 \mathrm{~K}$ span the temperature range from the quasi-rigid case to full motional averaging of the intramolecular dipolar couplings.

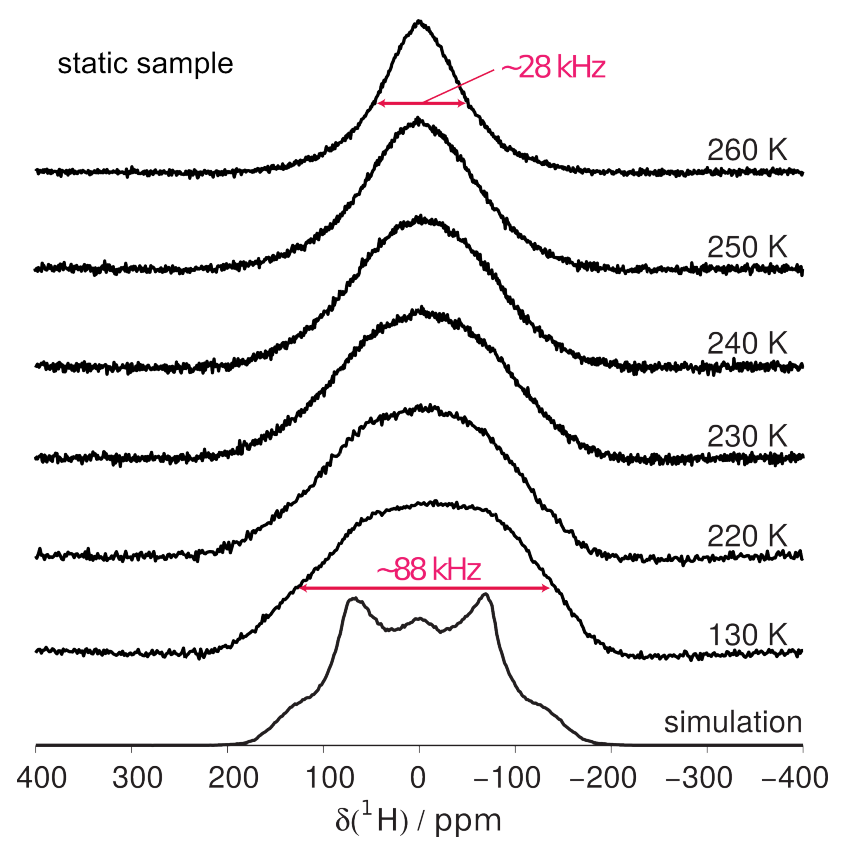

Fig. 2: Single-pulse spectra of hexagonal ice in a pure water sample at various temperatures without MAS in 
comparison with a ten-spin simulation. The line narrowing with increasing temperature is attributed to dynamics. Spectra consist of a single scan, acquired after a waiting time of at least $12 \mathrm{~h}$ to guarantee thermal equilibration (and spin relaxation).

The ice spectra of a pure water sample under $40 \mathrm{kHz}$ MAS are shown in Figure 3a. Spectra were acquired as a single scan after typically 12 hours (much longer than $5^{*} \mathrm{~T}_{1}$ but needed for thermal equilibration). At the lowest temperature of $100 \mathrm{~K}$ the MAS narrows the spectrum and a side-band manifold with a line width of the center band of approximately $3.6 \mathrm{kHz}$ is observed, compared to a line with of over $80 \mathrm{kHz}$ in the static case. The spectrum remains mostly unchanged up to a temperature of $222 \mathrm{~K}$. Starting from $230 \mathrm{~K}$ the central line begins to broaden and the sidebands start to decrease. At the highest measured temperature of $273 \mathrm{~K}$ the line width has increased to a value of almost $10 \mathrm{kHz}$ and the side bands are no longer visible.

Very similar spectra were obtained for ice in the HET-s (218-289) protein sample, Figure 3b. The ice spectrum was separated from the protein and water spectrum by recording the spectra after a waiting time of typically 12 hours and subtracting one spectrum taken after a saturation followed by only $3 \mathrm{~s}$ of waiting time which is long enough to relax the protein and water components. $T_{1}$ relaxation times were determined by saturation recovery and yielded $2000 \mathrm{~s}$ at $240 \mathrm{~K}$ for the slowly relaxing ice component and $0.5 \mathrm{~s}$ for the fast component containing the water and protein signals (see Figure 4). The large difference in $T_{1}$ times indicates that no efficient polarization transfer takes place between water and ice while exchange on the millisecond time scale was observed between HET-s(218-289) and liquid water (van Melckebeke et al. 2011). This behaviour could be explained if the interface between ice and water would not allow for polarization transfer, which is very unlikely due to the dynamical nature of the phase boundary with chemical-exchange between water molecules in ice and water. Another more likely explanation might be the size of the ice particles, being much larger than the HET-s (218-289) fibril. From the spin-diffusion rate constant that can be estimated to be $7 \cdot 10^{-}$ ${ }^{17} \mathrm{~m}^{2} / \mathrm{s}$, following the perturbation Ansatz of Abragam (Abragam 1961)(see Materials and Methods) and neglecting the effect of MAS, we can roughly estimate that the diameter of ice particles (in their smallest dimension) should exceed $2 \mu \mathrm{m}$, while the diameter of the HET-s (218-289) fibrils is roughly $3 \mathrm{~nm}$ (see structure $2 \mathrm{RMN}$ in the protein data base). Ice crystals must therefore to some degree displace and 
concentrate the protein during the freezing process.

a)

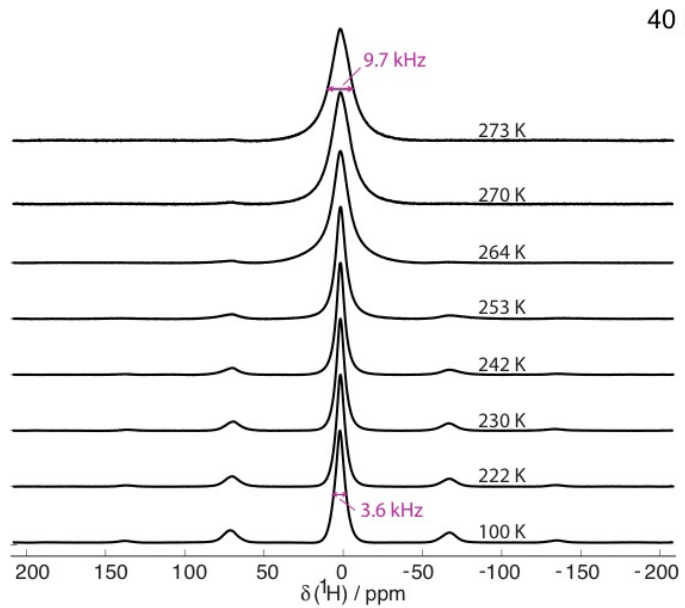

$40 \mathrm{kHz}$ MAS

Fig. 3: Single-pulse spectra of ice acquired in a pure water sample a) and a fully deuterated back exchanged HET-s (218 -289) sample b) at various temperatures and an MAS frequency of $40 \mathrm{kHz}$. Spectra are plotted to have the same maximum intensity. Spectra consist of a single scan, acquired after a waiting time of $12 \mathrm{~h}$ to guarantee thermal equilibration (and spin relaxation) and, in b) a further scan was acquired $3 \mathrm{~s}$ later to subtract the fast-relaxing component. The two scans are individually shown in Figure S1.

In the case of temperatures between $100 \mathrm{~K}$ and $222 \mathrm{~K}$, for pure ice or ice in the protein sample, the rateconstant of the jump processes is lower than the MAS frequency, and coherent averaging is relatively efficient. The remaining line width is given by the homogenous nature of the dipole interaction (Maricq and Waugh 1979) and is still more than 100 times larger than the liquid water component at $40 \mathrm{kHz}$ MAS. The ice resonance further broadens towards higher temperatures. The successive broadening originates from an interference with the molecular dynamic approaching a rate constant of $80 \mathrm{kHz}$. At temperatures approaching $273 \mathrm{~K}$, the dipolar line width, the MAS frequency and the exchange rate constant are then all on the same order of magnitude (Barnaal and Lowe 1968). This interference leads to a further broadening of the ice resonance to about $17 \mathrm{ppm}$. Therefore, MAS does not lead to a narrow ice proton line close to the freezing point. 

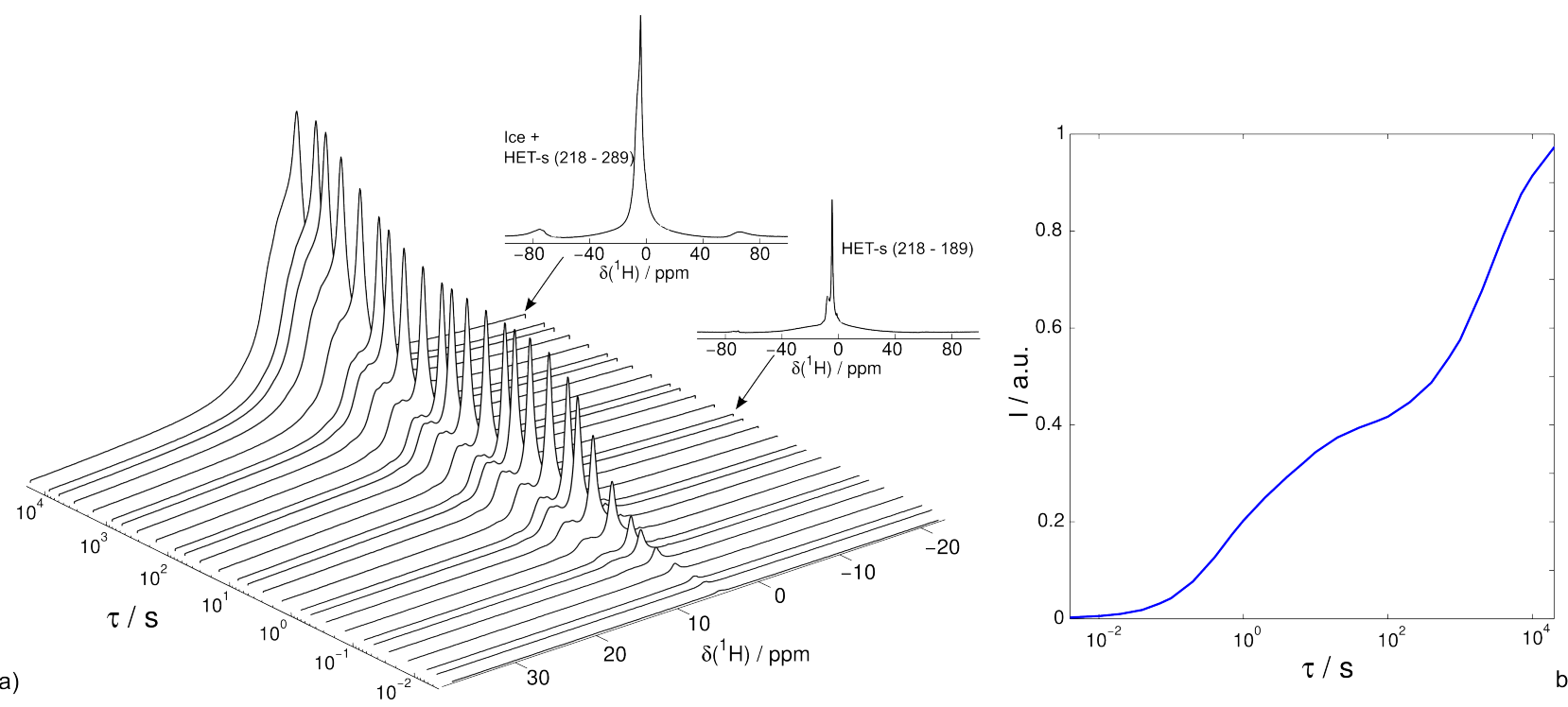

Fig. 4: a) Saturation recovery build-up of proton signal in deuterated back exchanged HET-s (218-289). The relaxation times of the protein and mobile water and ice differ by roughly four orders of magnitude (2000 s vs. $0.5 \mathrm{~s}$ ), visible by the double exponential rise of the center peak as shown in. b)

Alternatively to MAS one may try to narrow the ice line via a homonuclear decoupling sequence, e.g., MSHOT3 (Hohwy and Nielsen 1997). Averaging the dipolar interactions will also lead to a scaling of the proton chemical-shift tensor (scaling factor of 0.35). Figure 5a shows the homonuclear decoupled spectra of ice for temperatures between $185 \mathrm{~K}$ and $260 \mathrm{~K}$. At the lowest temperature of $185 \mathrm{~K}$ one can clearly see the CSA tensor of ice as reported in (Burum and Rhim 1979; Rhim et al. 1979). Once the temperature increases the CSA tensor starts to become gradually averaged by the defect motion. The line gets narrower and more symmetrical until a temperature of $245 \mathrm{~K}$. The same observations can be made for ice observed in fully deuterated and back exchanged HET-s(218-289), which is shown in Figure 5b, again indicating that ice in the biological sample is rather pure and undisturbed by the presence of protein. Above the line broadens again due to interference between the proton jumps and the decoupling frequency as seen in the GAMMA simulation of MSHOT-3 on a dynamic two-spin system with full CSA and dipolar coupling shown in Figure 5c. As in the case of MAS, multiple-pulse decoupling is not able to efficiently narrow the proton lines at temperatures slightly below freezing. The observed lineshapes allow for a semi-quantitative determination of the defect jump rate. 


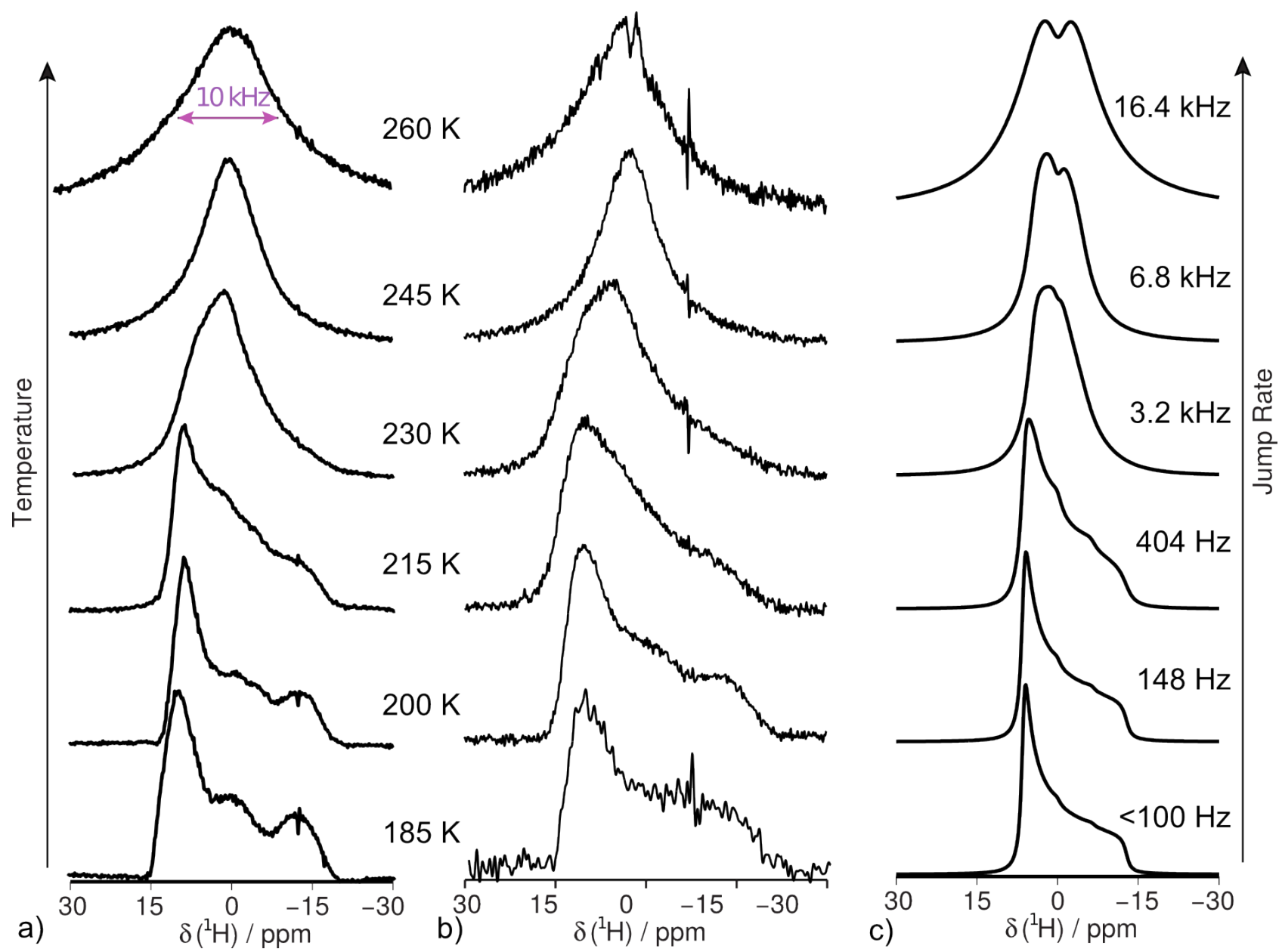

Fig. 5: a) Windowed MSHOT-3 spectra of pure ice. b) Windowed MSHOT-3 spectra of ice in deuterated back exchanged HET-s(218-289). c) Simulation of MSHOT-3 that best fits the experimental spectrum, on a water molecule with CSA and full intramolecular dipolar coupling. Spectra consist of a single scan, acquired after a waiting time of $12 \mathrm{~h}$ to guarantee thermal equilibration (and spin relaxation) and, in $\mathbf{b}$ ) a further scan was acquired $3 \mathrm{~s}$ later to subtract the fast-relaxing component. The two scans are separately shown in Figure S2.

A more complete averaging of the dipolar interaction can be obtained by MAS on isotopically diluted samples where the rest protons in a deuterated sample are observed. Figure 6 shows the proton spectra of deuterated ice at various temperatures for MAS at $10 \mathrm{kHz}$. The line width reduces to about $380 \mathrm{~Hz}$ due to the sparse amount of and therefore weaker interactions amongst the remaining protons in the sample as well as the MAS. The line appears slightly asymmetric which can be attributed to field homogeneity changes upon cooling. With increasing temperature, the line broadens due to the internal dynamics until a temperature of approximately $240 \mathrm{~K}$ with a line width of about $1.3 \mathrm{kHz}$. With a further increase in the temperature the line 
seems to start narrowing again, to a value of $960 \mathrm{~Hz}$ at $270 \mathrm{~K}$, which can be attributed to the jump rate leaving the regime of interference with the MAS.

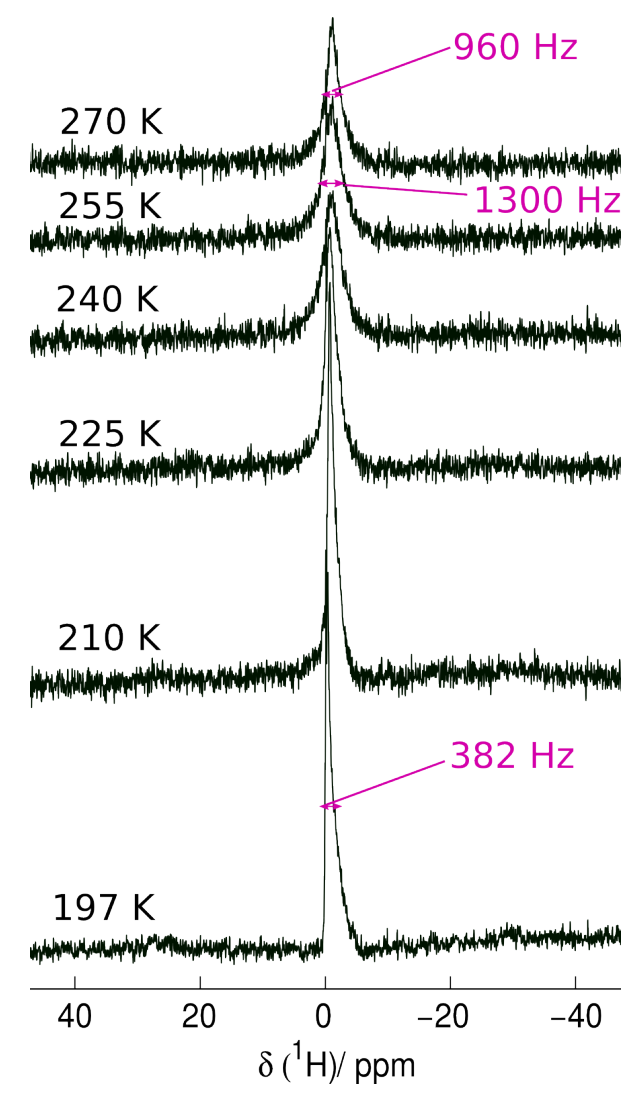

Fig. 6: Spectra of remaining protons in $99.8 \% \mathrm{D}_{2} \mathrm{O}$ at $10 \mathrm{kHz}$ MAS. Spectra consist of a single scan, acquired after a waiting time of at least $12 \mathrm{~h}$ to guarantee thermal equilibration (and spin relaxation).

Figure 7 gives a comparison of the jump rates obtained from the simulation of the MSHOT-3 experiment of Figure 5 and previous results from longitudinal relaxation (Barnaal and Lowe 1968) and ${ }^{17} \mathrm{O}$ quadrupolar line shape analysis (Ba et al. 2011) on samples that are purer than our pure water. Despite the known dependence of the rate constant on impurity concentration (Smyth and Hitchcock 1932; Fujara et al. 1988) the previous data sets coincide reasonably well with systematically faster values for our less pure samples. We note that the pure water ice and the ice crystals within the HET-s(218-289) sample behave, within error, identically. 


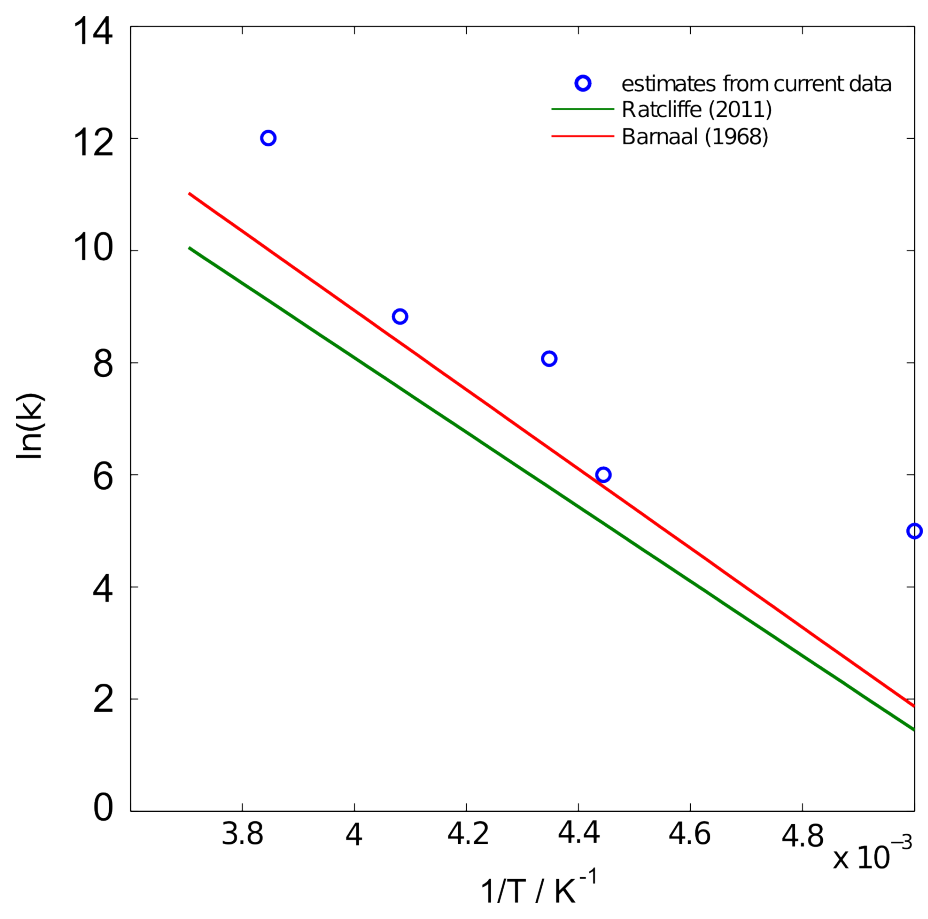

Fig. 7: Comparison of literature results for jump rates in ice and the values gained from an estimated match between MSHOT-3 experiments and simulation in GAMMA (blue circles). Within our experimental precision, the pure water and water in HET-s(218-289) coincide. The solid lines represent the linear regressions using the data points in (Ba et al. 2011) (green) and (Barnaal and Lowe 1968) (red).

To investigate the presence of polarization transfer between the ice and water phases in the HET-s(218-289) sample, saturation-transfer (CEST) experiments were performed (Fig. 8): the intensity-changes of the water resonance line in a $90^{\circ}$ pulse- detection experiment was monitored after a presaturation period with proton irradiation. The spectrum is recorded as a function of the irradiation frequency for proton irradiation of 800 ms with an rf field strength of $400 \mathrm{~Hz}$. At $280 \mathrm{~K}$, the water and protein spectra are reproduced in the CEST experiments due to the fast polarization transfer through chemical exchange between protein and water. The exchange proceeds via the sidechain exchangeable protons but is distributed via spin diffusion throughout the protein protons(van Melckebeke et al. 2011). At subfreezing temperatures, the CEST spectra become broad, tracing out the ice spectrum and indicating polarization transfer between ice and water on a timescale of milliseconds to seconds. We interpret this as polarization transfer between $\mathrm{H}_{2} \mathrm{O}$ protons near the surface of the ice particles and the water-ice system which equilibrates on a faster timescale. As indicated by the large difference in $T_{1}$ between water/protein and ice, there is no polarization transfer between a large fraction of 
the ice phase with water/protein but the high sensitivity of the CEST experiments allows to detect the exchange between the surface of the ice particles and water/protein.

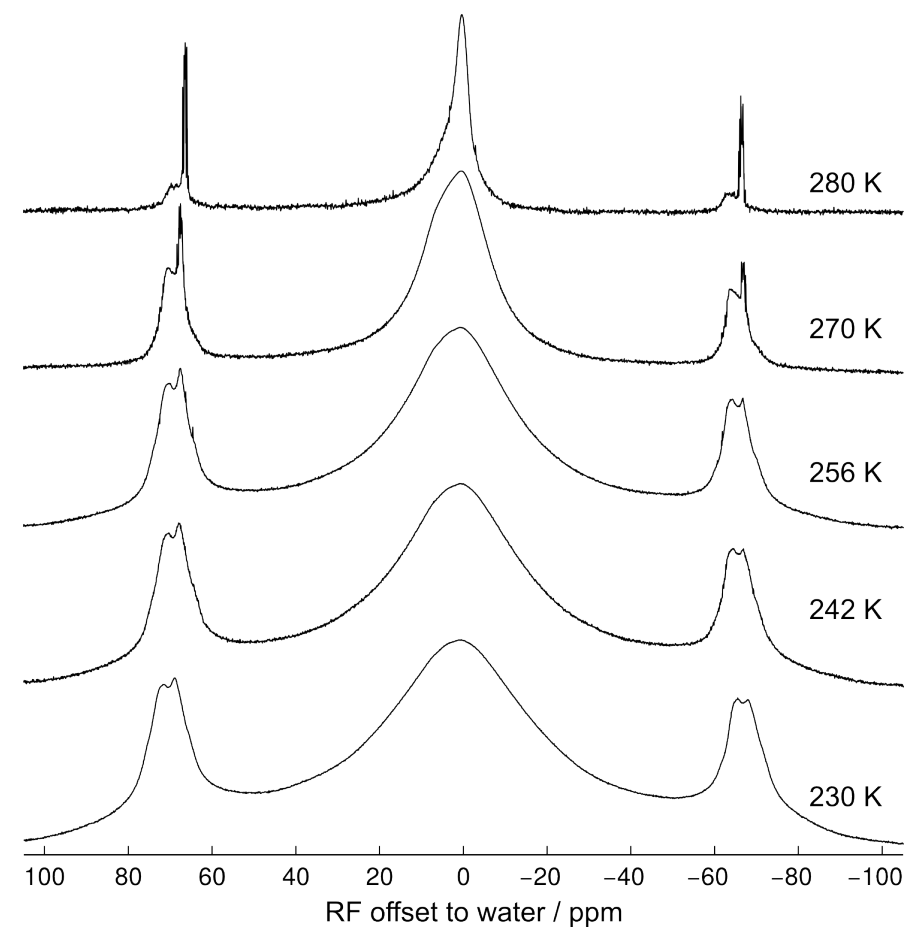

Fig. 8: Saturation-transfer (CEST) experiments detected on the water signal of HET-s(218-289). Above freezing, polarization transfer between water and protein is seen in the spectrum as well as the spinning sidebands, below freezing the broad polarization-transfer profiles indicate polarization transfer between ice and water/protein.

\section{Conclusion}

We have recorded spectra of ice in a protein (frozen HET-s(218 -289)) and have found them to be largely identical to the ones of pure water ice. The spectra are strongly influenced by the reorientational motion of the water molecules in ice which interferes with coherent line narrowing by MAS or homonuclear decoupling. The interference is most pronounced between $200 \mathrm{~K}$ and the melting point. The very long $\mathrm{T}_{1}$ relaxation times of $2000 \mathrm{~s}$ found for ice in frozen HET-s $(218-289)$ indicate that the ice crystals in the biological system are larger than the spin diffusion length on the timescale of the $\mathrm{T}_{1}$ times and that the crystals are therefore larger 
than $2 \mu \mathrm{m}$ in the shortest dimension, three orders of magnitude larger than the HET-s (218-289) fibril.

Freezing thus likely leads to a displacement of protein fibrils and a local concentration of the sample.

\section{Acknowledgements}

We thank P. K. Madhu for interesting discussions on homonuclear decoupling. This work has been supported by the Swiss National Science Foundation (Grant 200020_159707 and 200020_146757), the ETH Zurich Research Grant (ETH-08 10-1) and the French ANR (ANR-12-BS08- 0013-01, ANR-14-CE09-0024B.

\section{References}

Abragam A (1961) The Principles of Nuclear Magnetism. Oxford Science Publications

Akbey U, Oschkinat H (2016) Structural biology applications of solid state MAS DNP NMR. J Magn Reson 269:213-224. doi: 10.1016/j.jmr.2016.04.003

Ba Y, Ripmeester JA, Ratcliffe Cl (2011) Water molecular reorientation in ice and tetrahydrofuran clathrate hydrate from lineshape analysis of 170 spin-echo NMR spectra. Canadian Journal of Chemistry 89:1055-1064. doi: 10.1139/v11-040

Bagchi B (2013) Water in Biological and Chemical Processes: From Structure and Dynamics to Funct. Cambridge University Press

Barnaal DE, Lowe I (1968) Proton Spin-Lattice Relaxation in Hexagonal Ice. J Chem Phys 48:4614-6. doi: $10.1063 / 1.1668036$

Barnes AB, Paëpe GD, van der Wel PCA, et al (2008) High-Field Dynamic Nuclear Polarization for Solid and Solution Biological NMR. Appl Magn Reson 34:237-263. doi: 10.1007/s00723-008-0129-1

Bauer T, Dotta C, Balacescu L, et al. Line-Broadening in Low-Temperature Solid-State NMR Spectra of Fibrils. submitted for publication.

Bernal JD, Fowler RH (1933) A Theory of Water and Ionic Solution, with Particular Reference to Hydrogen and Hydroxyl Ions. J Chem Phys 1:515-35. doi: 10.1063/1.1749327

Bertini I, Luchinat C, Parigi G, et al (2011) Solid-state NMR of proteins sedimented by ultracentrifugation. P Natl Acad Sci USA 108:10396-10399. doi: 10.1073/pnas.1103854108

Böckmann A, Emsley L, Galinier A, et al (2005) Water-protein hydrogen exchange in the micro-crystalline protein Crh as observed by solid state NMR spectroscopy. J Biomol NMR 32:195-207. doi: 10.1007/s10858-005-8073-y

Böckmann A, Ernst M, Meier BH (2015) Spinning proteins, the faster, the better? J Magn Reson 253:71-79. doi: 10.1016/j.jmr.2015.01.012

Böckmann A, Gardiennet C, Verel R, et al (2009) Characterization of different water pools in solid-state 
NMR protein samples. J Biomol NMR 45:319-327. doi: 10.1007/s10858-009-9374-3

Burum DP, Rhim W-K (1979) An improved NMR technique for homonuclear dipolar decoupling in solids: Application to polycrystalline ice. J Chem Phys 70:3553. doi: 10.1063/1.437892

Can TV, Ni QZ, Griffin RG (2015) Mechanisms of dynamic nuclear polarization in insulating solids. J Magn Reson 253:23-35. doi: 10.1016/j.jmr.2015.02.005

Fujara F, Wefing S, Kuhs WF (1988) Direct observation of tetrahedral hydrogen jumps in ice Ih. J Chem Phys 88:6801-6809. doi: 10.1063/1.454426

Geil B, Kirschgen TM, Fujara F (2005) Mechanism of proton transport in hexagonal ice. Phys Rev B 72:014304. doi: 10.1103/PhysRevB.72.014304

Havlin R, Tycko R (2005) Probing site-specific conformational distributions in protein folding with solid-state NMR. P Natl Acad Sci USA 102:3284-3289. doi: 10.1073/pnas.0406130102

Hohwy M, Nielsen NC (1997) Elimination of high order terms in multiple pulse nuclear magnetic resonance spectroscopy: Application to homonuclear decoupling in solids. J Chem Phys 106:7571-7586.

Jeffrey GA, Saenger W (1994) Hydrogen bonding in biological structures. Springer-Verlag

Lesage A, Böckmann A (2003) Water-protein interactions in microcrystalline Crh measured by H-1-C-13 solid-state NMR spectroscopy. J Am Chem Soc 125:13336-13337. doi: 10.1021/ja036720y

Lesage A, Gardiennet C, Loquet A, et al (2008) Polarization transfer over the water-protein interface in solids. Angew Chem Int Ed Engl 47:5851-5854. doi: 10.1002/anie.200801110

Lynden-Bell R, Moris SC, Barrow JD, et al (2010) Water and Life: The Unique Properties of H2O. CRC Press

Maricq M, Waugh J (1979) NMR in rotating solids. J Chem Phys 70:3300.

Rhim W-K, Burum DP, Elleman DD (1979) Proton anisotropic chemical shift spectra in a single crystal of hexagonal ice. J Chem Phys 71:3139-3141. doi: 10.1063/1.438664

Siegle DG, Weithase M (1969) Spin-Gitter-Relaxation der Protonen in hexagonalem Eis. Z Physik 219:364380.

Siemer AB, Huang K-Y, McDermott AE (2010) Protein-ice interaction of an antifreeze protein observed with solid-state NMR. P Natl Acad Sci USA 107:17580-17585. doi: 10.1073/pnas.1009369107

Siemer AB, Huang KY, McDermott AE (2012) Protein Linewidth and Solvent Dynamics in Frozen Solution NMR. PLoS ONE. doi: 10.1371/journal.pone.0047242.g001

Siemer AB, McDermott AE (2008) Solid-State NMR on a Type III Antifreeze Protein in the Presence of Ice. J Am Chem Soc 130:17394-17399. doi: 10.1021/ja8047893

Slichter C (1978) Principles of Magnetic Resonance.

Smith S, Levante T, Meier BH, Ernst R (1994) Computer Simulations in Magnetic Resonance: An Object Oriented Programming Approach. J Magn Reson Ser A 106:75-105.

Smyth CP, Hitchcock CS (1932) Dipole Rotation in Crystalline Solids. J Am Chem Soc 54:4631-4647. doi: 10.1021/ja01351a020

van Melckebeke H, Schanda P, Gath J, et al (2011) Probing water accessibility in HET-s(218-289) amyloid 
fibrils by solid-state NMR. J Mol Biol 405:765-772. doi: 10.1016/j.jmb.2010.11.004

van Melckebeke H, Wasmer C, Lange A, et al (2010) Atomic-resolution three-dimensional structure of HETs(218-289) amyloid fibrils by solid-state NMR spectroscopy. J Am Chem Soc 132:13765-13775. doi: $10.1021 / j a 104213 \mathrm{j}$

van Vleck J (1948) The dipolar broadening of magnetic resonance lines in crystals. Phys Rev 74:1168.

Wasmer C, Lange A, van Melckebeke H, et al (2008) Amyloid fibrils of the HET-s(218-289) prion form a beta solenoid with a triangular hydrophobic core. Science 319:1523-1526. doi: 10.1126/science.1151839

Wasmer C, Benkemoun L, Sabate R, et al (2009a) Solid-state NMR spectroscopy reveals that E. coli inclusion bodies of HET-s(218-289) are amyloids. Angew Chem Int Ed Engl 48:4858-4860. doi:

10.1002/anie. 200806100

Wasmer C, Schütz A, Loquet A, et al (2009b) The molecular organization of the fungal prion HET-s in its amyloid form. J Mol Biol 394:119-127. doi: 10.1016/j.jmb.2009.09.015 